Check for updates

Cite this: Phys. Chem. Chem. Phys., 2017, 19, 23878

\section{Formation of titanium dioxide core-shell microcapsules through a binary-phase spray technique}

\author{
Jonatan Bergek, Björn Elgh, Anders E. C. Palmqvist and Lars Nordstierna (D) *
}

\begin{abstract}
Core-shell microcapsules consisting of a titanium dioxide shell and a hydrophobic solvent core have been prepared with diameters of a few micrometers and a narrow size distribution using a simple and fast airbrush technique. These microcapsules were prepared at room temperature in a single-step process in which an oil with a dissolved titanium alkoxide precursor was forced together with an aqueous solution, containing a surface-active polymer, through a narrow spray nozzle using a nitrogen gas propellant. Several different parameters of chemical, physical, and processing origin were investigated to find an optimal recipe. Two different alkanes, one ketone, and four alcohols were tested and evaluated as core materials, alone or together with the antifungal biocide 2-n-octyl-4-isothiazolin-3-one (OIT). Long-chain alcohols were found suitable as core oil due to their low solubility in water and surface activity. The addition of the surface-active polymers in the water phase was important in aiding the formation and stabilization of the titanium dioxide shell. An impressive loading of 50 wt\% of the semi-hydrophobic OIT was possible to encapsulate using this simple and applicable procedure.
\end{abstract}

Received 19th April 2017, Accepted 31st July 2017 DOI: $10.1039 / \mathrm{c7cp} 02571 \mathrm{~h}$ rsc.li/pccp

\section{Introduction}

Microencapsulation is the general term for methods where small (1-1000 $\mu \mathrm{m})$ solid particles, liquid droplets, or gas bubbles are coated with a thin layer of coating or a shell material. ${ }^{1}$ There are numerous methodological approaches and technical strategies to formulate microcapsules and the methods can be classified as chemical, physical, or mechanical routes or combinations of these. Chemical processes include different types of polymerization layer growth such as interfacial, emulsion, suspension, or chain growth. The physical processes based on coacervation/ phase separation are the broadest class of encapsulation methods. Mechanical processes are e.g. spray-drying and electrostatic encapsulation. ${ }^{1,2}$ Moreover, the use of microfluidic devices is an interesting approach, forming microcapsules with a narrow size distribution. ${ }^{3,4}$

Microcapsules are of interest in several different areas such as agriculture, cosmetics, textiles, coatings, and especially the pharmaceutical industry, for encapsulating, transporting and controlling the release of active substances..$^{2,3,5-7}$ The desirable time span considering the release of active ingredients out from the micro-container to a surrounding medium can be immediate, ${ }^{8}$ triggered ${ }^{9}$ or sustained. ${ }^{10}$ Therefore, the formulation strategy of

Department of Chemistry and Chemical Engineering, Chalmers University of Technology, 41296 Göteborg, Sweden. E-mail: lars.nordstierna@chalmers.se; Fax: +46 31 160062; Tel: +4631 7722973 the microcapsule is of crucial importance to be able to achieve a desirable release rate of the active substance to the surrounding medium. To overcome problems with premature release, especially concerning polymeric systems, the permeability of the shell material can be reduced either by kinetic or thermodynamic approaches. ${ }^{11}$ Kinetic approaches are most commonly used for reducing the diffusivity of an active molecule through the polymer shell, ${ }^{12}$ by e.g. using semi-crystalline polymers instead of amorphous, cross-linking the shell material or using nano-clays in the microparticle. ${ }^{12,13}$ A thermodynamic approach can be exemplified by the use of polyelectrolyte multilayers anchored to the shell, as a solubility barrier for releasing active substances. ${ }^{10,12}$

Microcapsules are often polymer-based, especially the shell, and polymers for this purpose can be e.g. poly(methyl metacrylate), polyester, poly(lactic- $\mathrm{co}$-glycolic acid) or poly(lactic acid). ${ }^{7,14,15}$ Although polymer systems have benefits with regard to their versatility in terms of colloid and interface chemistry one can also find drawbacks, often related to the soft matter structure, and long-term release over years is difficult to achieve. Examples are the effects of microscopic porosity, ${ }^{13,14}$ high burst release, ${ }^{12}$ polydisperse sizes and structures ${ }^{3}$ but also toxic and volatile solvents ${ }^{16}$ used in intermediate formulation steps.

When aiming for long-term sustained release or even close to no release from micro-reservoirs, applying an inorganic coating to the particle can effectively decrease the release rate. Examples in the literature describe the formulation of solid or core-shell particles that are subsequently coated with a metallic layer. ${ }^{17-19}$ 
Metal oxide shells can be another viable alternative to polymeric or composite barriers. Titanium dioxide, titania, is cheap, abundant, environmentally benign, and non-toxic. While polymeric barriers typically are synthesized or formulated using harsh organic solvents, a plethora of titania synthesis routes are known, many of which are waterborne or based on nonhazardous solvents. ${ }^{20}$ Furthermore, titania may be synthesized from both hydrophobic and hydrophilic precursors, ${ }^{21}$ enabling innovative emulsion and microemulsion reaction systems to be formulated.

There are reported ways to formulate core-shell microcapsules consisting of a titania shell, using spray techniques ${ }^{22,23}$ for hollow microcapsules or non-aqueous emulsions ${ }^{24,25}$ for hollow or filled microcapsules. As stated above, the use of microcapsules is often to control the release of an active ingredient to the surrounding. In every step of the chosen formulation route, the active ingredient should not undergo any unexpected changes, as e.g. thermal degradation, or be denaturized by organic solvents.

In this work, the focus has been to encapsulate the widely used antifungal biocide 2- $n$-octyl-4-isothiazolin-3-one, abbreviated OIT. OIT belongs to the isothiazolinones, which are heterocyclic aromatic compounds. It is semi-hydrophobic, and often commercially used in the outdoor paint industry, but also in leather and cardboard products, as well as in polymeric materials such as PVC. $^{26,27}$ In previous studies, we have encapsulated OIT in homogenous polymer microspheres following the internal phase separation method, investigating the role of porosity ${ }^{13}$ in the release of biocide, as well as using superhydrophilic polyelectrolyte multilayers as a rate-determining barrier for diffusion. $^{10}$

Since titania is a commonly used pigment, and as we have shown in previous studies that encapsulation of OIT makes an advantageous impact from an applied perspective, i.e. sustained release from coatings, we believe that titania can be a well-suited shell material when aiming for controlled release of OIT from a core-shell microcapsule embedded in a paint matrix while serving as both an extended pigment and antifoulant. We here demonstrate a simple and fast microencapsulation route at room temperature, not including any toxic solvents or bi-products, using a spray nozzle method without intermediate steps.
The product is a well-defined core-shell microcapsule consisting of a titania shell surrounding a hydrophobic core of a long-chain fatty alcohol. We show that the selected setup can be used to encapsulate the semi-hydrophobic biocide OIT in the core, up to $50 \mathrm{wt} \%$.

\section{Experimental section}

The following chemicals were used as received; 2 - $n$-octyl-4isothiazolin-3-one (OIT, TCI Europe, $>98 \%$ ), titanium(Iv) butoxide (Sigma-Aldrich, 97\%), titanium(Iv) ethoxide (Sigma-Aldrich), titanium(Iv) 2-ethylhexyloxide (Sigma-Aldrich, 95\%), hexadecane (Sigma-Aldrich, 99\%), dodecane (Acros Organics, 99\%), cycloheptanone (Acros Organics, 98\%), 1-butanol (Fluka Chemika, $>98 \%$ ), 1-hexanol (Fluka Chemika, >98\%), 1-undecanol (Aldrich, 99\%), oleyl alcohol (Fluka Chemika, 50-60\%), nitrogen gas $\left(\mathrm{N}_{2}\right.$, AGA Gas), chloroform-d $\left(\mathrm{CDCl}_{3}\right.$, Aldrich, 99.8\%), poly(vinyl alcohol) (PVA, 95\% hydrolyzed, $M_{\mathrm{w}} 95000$, Acros Organics), poly(methacrylic acid) ( $M_{\mathrm{w}} 100000$, Polysciences). The water used was de-ionized using Milli- ${ }^{\mathrm{TM}}$ equipment and had a conductivity of $<0.055 \mathrm{mS} \mathrm{cm}^{-1}$.

The microcapsules were formed via a direct process, schematically illustrated in Fig. 1. An oil phase, consisting of a titania precursor and a biocide both dissolved in the so-called core oil, was mixed thoroughly for a few minutes before it was added to one of the two containers connected to an airbrush pen (A470 Airbrush, Áztek, USA). The containers connect to the airbrush pen via a thin tube each, one from each side of the device. The tubes lead the phases individually to a small compartment in the direct vicinity of the spray nozzle. The propellant gas connects to the rear part of the compartment and presses the content from the containers to the front, and through the nozzle. Different titanium alkoxide substances, different core oils, and the fraction of each component (including the biocide OIT) were examined in order to find optimal conditions. In the second airbrush container a water phase was added, consisting of either only water or a surface-active polymer.

The principal recipe after optimization was as follows with zero or $10 \mathrm{wt} \%$ of OIT encapsulated. The oil phase consisted of $0.35 \mathrm{~g}$ oleyl alcohol, $0.1 \mathrm{~g} \mathrm{Ti}(\mathrm{OBu})_{4}$ and alternatively $0.05 \mathrm{~g}$ OIT,
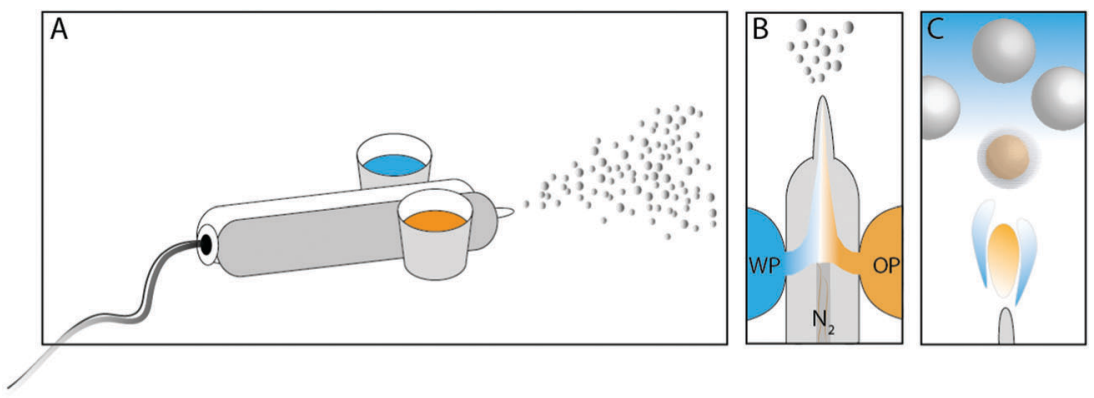

Fig. 1 (A) The airbrush setup. Nitrogen gas $\left(\mathrm{N}_{2}\right)$ is used as a propellant. (B) One container is filled with oil phase (OP) and the other container is filled with water phase (WP), and the two solutions are forced out together through the small spray nozzle. (C) The formation of core-shell microcapsules initiates in the direct vicinity of the spray nozzle, when the titanium precursor in the oil droplets comes in contact with surrounding water, and starts to form titanium dioxide in the interface between water and oil. The final product is microcapsules suspended in water phase. 
and was mixed before addition to the airbrush. The water phase consisted of $1 \mathrm{wt} \%$ PVA, and was in volumetric excess compared to the oil phase, typically $25 \mathrm{ml}$. The airbrush was propelled using nitrogen gas of 2.8 bar pressure and the spray nozzle was $0.70 \mathrm{~mm}$ in size, as stated by the manufacturer.

Immediately after filling the two containers with the oil phase and the water phase, respectively, spraying was started, and microcapsules were formed. In order to collect the sample, the horizontally aligned nozzle was aimed against a vertically aligned aluminum surface from where the formed capsules were easily rinsed down to a beaker, without additional support. The nozzle was approximately 20 centimeters away from the surface. However, the significance of the distance has been investigated without finding any immediate differences in the end result with respect to microencapsulation yield and the size distribution.

The collected suspension was then filtered through a 120 mesh, a width of $0.125 \mathrm{~mm}$, (AB Derma, Sweden) of stainless steel to separate a minor fraction of larger aggregates consisting of a mixture of the unreacted oil phase material and the reacted precursor. The remaining suspension, consisting of microcapsules in the $1 \mathrm{wt} \%$ PVA water phase, was stored for further analyses.

A small amount of each produced batch was centrifuged (VWR, USA) in order to quickly analyze if the sample contained particles of the core-shell structure or just $\mathrm{TiO}_{2}$-particles, or both. Creaming of particles occurs by centrifugation if oil is encapsulated within a titania shell while sedimentation indicates particles only consisting of titania.

The size distribution and the shape of the microcapsules were analyzed using optical microscopes (Axio Scope.A1 and Axio Imager Z2m, Zeiss, Germany), equipped with a digital camera system (AxioCam MRC5 and AxioCam 506 color, Zeiss, Germany). By collecting several optical micrographs from the different suspensions, and using the Java-based image processing program ImageJ (National Institutes of Health, USA) to measure the size of the microcapsules, histograms of the size distribution were achieved followed by the calculation of the log-normal size distribution.

A fraction of the suspension from the principal recipe was analyzed using UV/vis spectrophotometry and NMR spectroscopy in order to determine the yield of microencapsulation. Duplicate suspension samples of precise weight were dried in an incubator at $60{ }^{\circ} \mathrm{C}$ for 20 hours to remove water (and the possible presence of produced 1-butanol). Thereafter, $3 \mathrm{ml}$ chloroform was added to one of the dried samples for further UV studies, while $1 \mathrm{ml} \mathrm{CDCl}$ was added to the second sample for further NMR studies. The samples were vigorously shaken to dissolve the content of oleyl alcohol and OIT in the added solvent. The solution for UV-analysis was filtered using a syringe with a fitted filter (Pall Acrodisc $0.2 \mu \mathrm{m}$ Fluorodyne II membrane $25 \mathrm{~mm}$, USA). The concentration of OIT in the filtrate was then determined using a UV/vis spectrophotometer (Agilent 8453, USA), at a wavelength of $276 \mathrm{~nm}$ as previously described. ${ }^{13}$ A $650 \mu$ volume of the deuterated solution was transferred to a $5 \mathrm{~mm}$ NMR tube. Another NMR tube contained a reference $\mathrm{CDCl}_{3}$ solution with known amounts of 1-butanol, oleyl alcohol, and OIT. Quantitative ${ }^{1} \mathrm{H}$ NMR spectra were acquired using a Varian MR 400 spectrometer (Palo Alto, CA). Each spectrum was collected by 64 acquisitions using a relaxation delay of $2 \mathrm{~s}$ and a $45^{\circ}$ RF-pulse followed by a $3 \mathrm{~s}$ acquisition time with a $8013 \mathrm{~Hz}$ spectral width in the frequency domain. The experiments in this work were carried out at $25{ }^{\circ} \mathrm{C}$. For the spectral analysis and integral calculations, the software program MestReNova version 8.1.2 (Mestrelab Research, Santiago de Compostela, Spain) was used.

\section{Results and discussion}

The specifically detailed spectroscopic characterization of the microcapsules from the so-called principal recipe was carried out to determine the encapsulation yield. The yield is here defined as the fraction of OIT leaving the airbrush container that ends up in micron-sized core-shell particles. It was found using UV/vis spectrophotometry that $35 \%$ of the OIT present in the starting oil phase was encapsulated in micron-sized coreshell particles of the final suspension. This result was confirmed using the NMR study which is presented in Fig. 2. Quantitative NMR spectroscopy comparing the spectra (Fig. 2a and b) between the reference sample and the suspension sample revealed a yield of $38 \%$ (compared to $35 \%$ obtained using UV/vis spectrophotometry). Using NMR, it could also be concluded (Fig. 2c at $0.88 \mathrm{ppm}$ ) that the OIT: oleyl alcohol ratio remained the same in the microcapsules as in the initial oil phase. Moreover, the spectroscopic resolution of NMR (Fig. $2 \mathrm{c}$ at $0.93 \mathrm{ppm}$ ) revealed that no 1-butanol was present in the sample of analysis, i.e. here evaporated during the drying step. Further detailed spectroscopic studies are needed to evaluate the reaction yield of the titanium precursor.

Several different parameters of chemical, physical, and processing origin were investigated to find an optimal recipe for the formation of titania core-shell microcapsules. We will here focus on the discussion from the perspective of the water phase, the precursor, the core material, the active biocide, and finally the spray nozzle.

\section{The water phase}

Several water phases were investigated with regard to the content of a non-ionic surface-active polymer. It was shown that the addition of pre-hydrolyzed (95-99\%) PVA or PMAA was important for the outcome, with regard to stability, even at a low concentration of $1 \mathrm{wt} \%$ (up to $5 \mathrm{wt} \%$ was investigated). Here, oleyl alcohol was used as a core material in the oil phase.

The surface activity of the additive generated a slightly more narrow size distribution of the microcapsules and also a lower mean average size, see Fig. 3a. In a parallel study, microscopy analysis showed that large $(>10 \mu \mathrm{m})$ capsules easily break early subsequently to fabrication, therefore a narrow size distribution and a rather small microcapsule size are desired (microscopy images in Fig. 4). A more important reason to use surface-active polymers is that the stability of the oil droplets in the formulation 

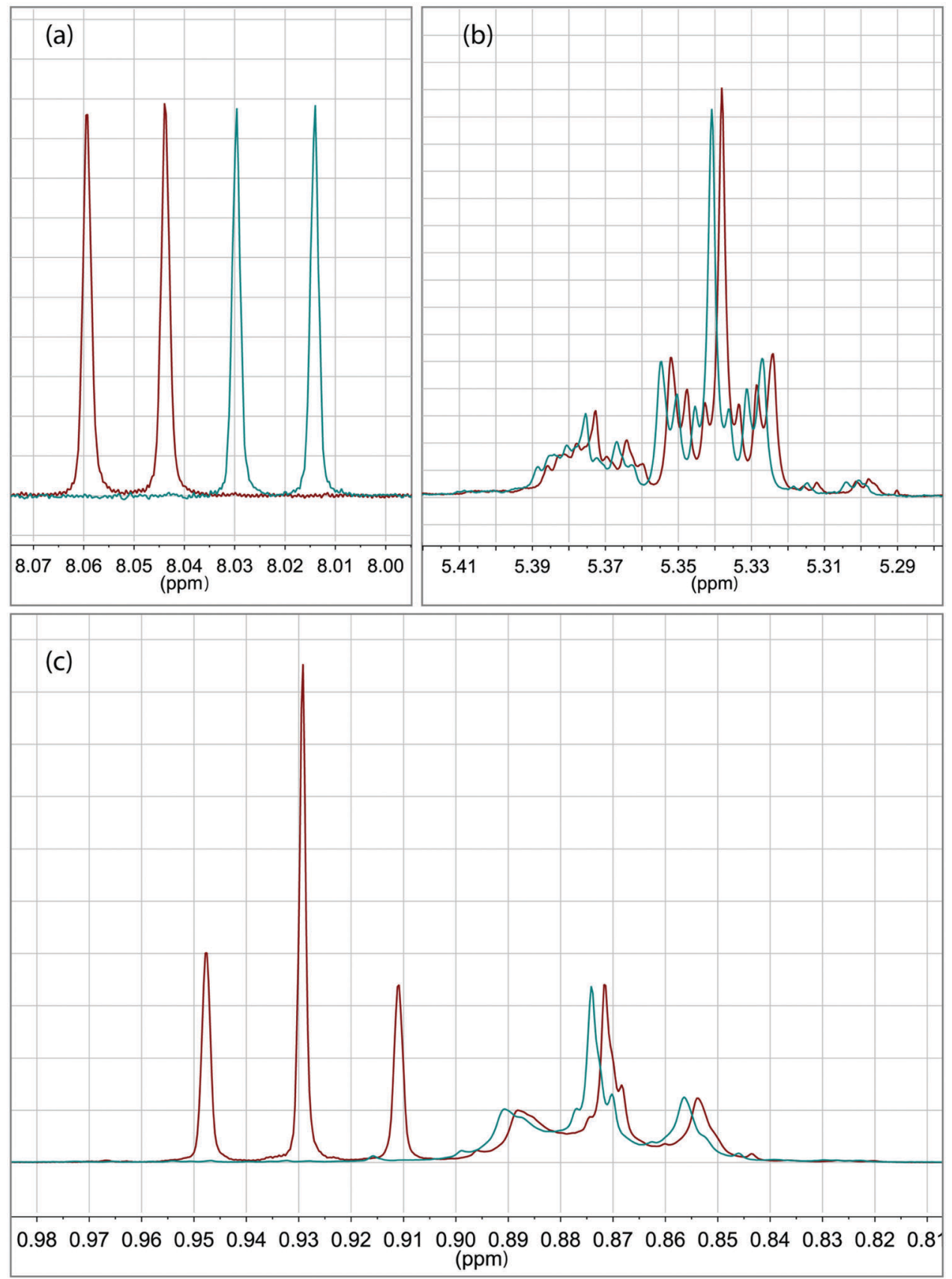

Fig. $2{ }^{1} \mathrm{H}$ NMR spectral regions of (a) aromatic OIT protons, (b) double-bond oleyl alcohol protons, and (c) methyl protons for $n$-butanol (centered at $0.93 \mathrm{ppm}$ ) and combined methyl protons for OIT and oleyl alcohol (centered at $0.88 \mathrm{ppm}$ ). Red spectrum represents the reference sample and turquoise spectrum represents the analyzed suspension prepared as described in the text. The red reference spectrum intensity is here normalized by 0.38 . The small deviation in the signal position with respect to the chemical shift is due to the sample difference of the butanol content.

setup has a great impact on the outcome of the microcapsule formation, which will be discussed more thoroughly at the end of this section. Microcapsules formed using only water were to a large extent broken after a few weeks in suspension, in contrast to when adding PVA or PMAA to the water phase. The stability of suspended microcapsules, loaded with pure oleyl alcohol and a water phase consisting of $1 \mathrm{wt} \% \mathrm{PVA}$, was measured in terms of obtained size distributions (Fig. 3b). Instability was concluded to be more substantial for larger microcapsules providing a size distribution shifting to lower values during one month of investigation. It should be emphasized that the absolute number of capsules decreased over time due to instability and all size distributions in Fig. 3 are normalized to unity here.

\section{The precursor}

Titanium alkoxides, $\mathrm{Ti}(\mathrm{OR})_{n}$, are highly susceptible to nucleophilic attack as illustrated in Fig. 5. Upon contact with water, 

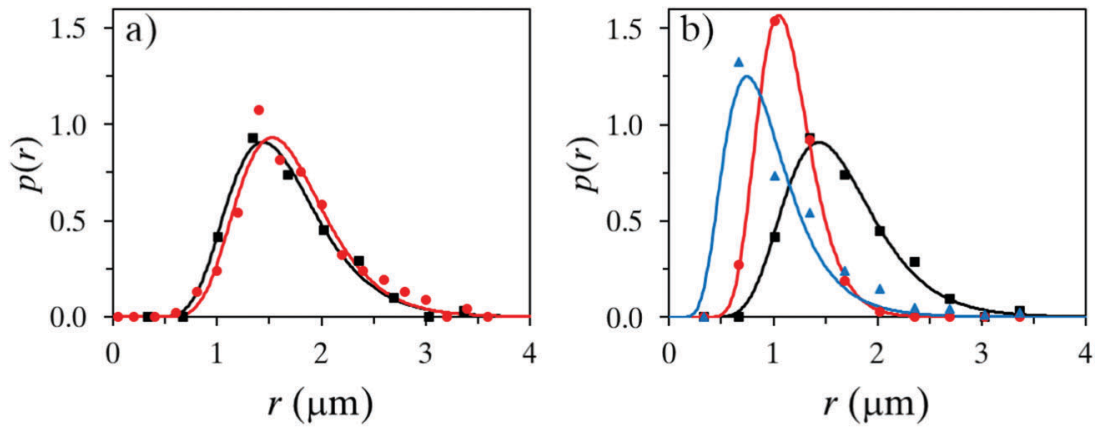

Fig. 3 (a) Size histograms and fitted lognormal distributions of microcapsules loaded with pure oleyl alcohol using 1 wt\% PVA in the water phase ( compared to using pure water (O). (b) Size histograms and fitted lognormal distributions of the microcapsules (1 wt\% PVA) directly after formulation ( $\mathbf{\square}$ ) one week after formulation ( $)$, and one month after formulation ( $\mathbf{\Lambda}$ ).

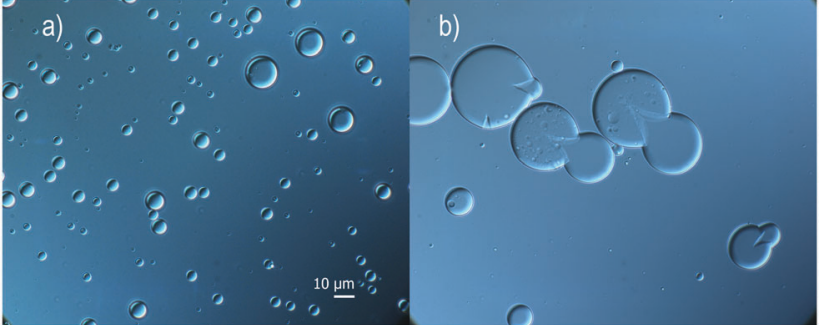

Fig. 4 (a) Optical micrograph of microcapsules loaded with oleyl alcohol using 1 wt\% PVA in water phase. (b) Optical micrograph of typical instability of larger microcapsule where broken and fractured microcapsules are shown with their inner core released out to the surrounding. Scale bar is valid for both micrographs.

titanium alkoxides are rapidly hydrolyzed via nucleophilic substitution resulting in the loss of a protonated alkoxy group. ${ }^{28}$ Condensation of the formed hydrolysis product may proceed via three different condensation reactions, alcoxolation, oxolation and olation. ${ }^{29}$ Alcoxolation proceeds via a nucleophilic attack resulting in the release of an alcohol. Oxolation and olation proceed via nucleophilic attack on water as the leaving group. As the hydrolysis of titanium alkoxide approaches completion, oxolation and olation become the predominating condensation reactions of the hydrolyzed titania species.

The oil phase consisted of a titania precursor, the core oil and optionally a biocide. Two criteria were followed in selecting components for the oil phase; the oil phase needed to be homogenously mixed, and no hydrolysis of the precursor should take place in the oil phase before addition to the synthesis setup. Three titania precursors were evaluated: titanium(rv) ethoxide, titanium(Iv) butoxide, and titanium(rv) 2-ethylhexyloxide. Due to the short alkoxy chains in titanium(Iv) ethoxide, this precursor reacts faster than the bulkier titanium(Iv) butoxide when in contact with water. In the setup used, it is important that the

Hydrolysis:

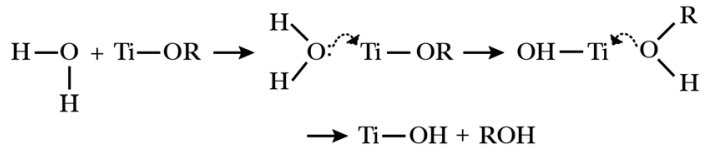

Alcoxolation:

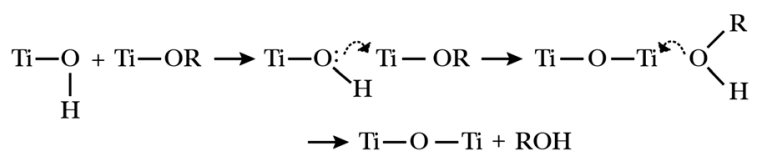

Oxolation:

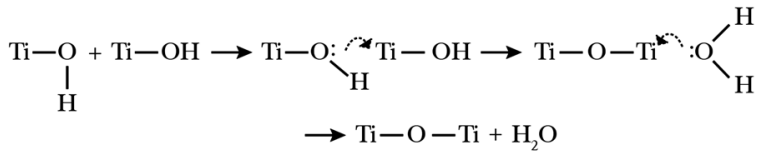

Olation:

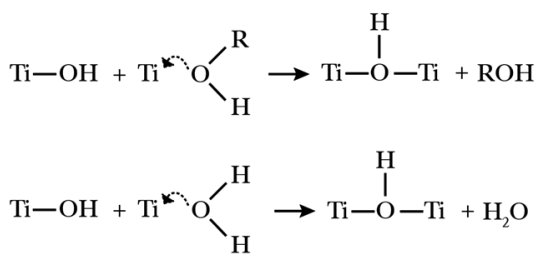

Fig. 5 Hydrolysis and condensation reactions leading to titanium dioxide formation. Curved arrows indicate nucleophilic attack and the transition state. 
hydrolysis of the precursor and the following condensation reactions do not occur too quickly, and especially that the condensation does not proceed too far before the mixture enters the spray nozzle. A too fast condensation will result in building up of titania deposits in the nozzle and eventually complete blocking of the nozzle. Based on initial trials we found titanium(Iv) butoxide and titanium(Iv) 2-ethylhexyloxide to be suitably reactive and the preferred alternatives when finding an optimal recipe, while titanium(Iv) ethoxide reacts too fast and prior to the nozzle outlet in this system. By analogous arguing, another possible precursor, $\mathrm{TiCl}_{4}$, was discarded due to its violent reaction with water.

\section{The oil phase}

Different liquid hydrophobic substances have been examined to constitute the oil phase in order to find a suitable core for the microcapsules. It was found that this selection is crucially important for the outcome. Two alkanes, hexadecane and dodecane, the ketone cycloheptanone, and four alcohols, 1-butanol, 1-hexanol, 1-undecanol and oleyl alcohol were tested, see Table 1. No capsules were formed by 1-hexanol nor by cycloheptanone, instead the precursor showed almost a direct reaction within the airbrush pen when in contact with the water phase, which made the addition of these substances inappropriate. 1-Butanol showed improved results in the setup and the oil phase here was not reacting with the water phase immediately, most probably due to slower kinetics by introduction of the hydrolysis product (1-butanol) on the right-hand side of the reaction scheme. However, only a small fraction of the oil phase ended up in microcapsules, a large fraction produced titania fragments or aggregations of large, broken capsules. Dodecane showed the same problems as 1-hexanol, forming an oil phase that reacted too fast, and therefore only formed a small number of microcapsules. Hexadecane improved the formation of microcapsules somewhat, but was still not an ideal candidate. A larger amount of the ingoing oil phase produced microcapsules, in size of a few microns, but also larger aggregates and flakes of titania were formed. As for 1-hexanol and dodecane, the oil phase consisting of hexadecane started to form titania early in the airbrush pen.

The core materials that were shown by far to be best suited in this setup were 1-undecanol and oleyl alcohol. These oil phases constituting a longer fatty alcohol produced a high yield of microcapsules and the nozzle flow of the oil phase and water phase,

Table 1 Two alkanes, one ketone, and four alcohols were examined as oil core materials. Water solubility and dynamic viscosity are given for each substance, as obtained from chemical databases ${ }^{30-34}$

\begin{tabular}{llc}
\hline Core material & Water solubility $\left(\mathrm{mg} \mathrm{l}^{-1}\right)$ & Dynamic viscosity $(\mathrm{cP})$ \\
\hline 1-Butanol & 63200 & 2.6 \\
Cycloheptanone & 35000 & 2.6 \\
1-Hexanol & 5900 & 4.6 \\
1-Undecanol & 8.0 & 14.7 \\
Oleyl alcohol & 0.05 & 35 \\
Dodecane & 0.0037 & 1.4 \\
Hexadecane & 0.00009 & 3
\end{tabular}

respectively, was not interrupted by the formation of titania in the interface between the two phases in an early stage of the experiment. From these results, it can be concluded that the core oil viscosity and the solubility of the core oil in water is of high importance. Both 1-undecanol and oleyl alcohol possess a low water solubility, while the viscosity is substantial compared to other oils of investigation.

In addition, another property of the long-chain alcohols compared to alkanes that may be beneficial in these experiments is their surface activity. ${ }^{31,35}$ As touched upon above concerning the use of a surface-active polymer in the water phase, the stabilization of the oil droplets in the excess of the water phase seems to aid the formation of the titania shell and improve the result. The use of long-chain alcohols may similarly be advantageous due to the surface activity. A hypothesis is that the formation of microcapsules with a titania shell surrounding an oil core starts immediately when entering the spray nozzle. The hydrolysis and subsequent condensation then occur inside the nozzle but might also continue directly after leaving the nozzle. If this step is ongoing for a short while after leaving the nozzle, the result relies on the stabilization of the oil droplets, including the presence of formed titania, since the shape of the droplet will decide the shape of the prepared microcapsule.

It could be seen as a disadvantage that the oil phase and the water phase need to follow the same channel to the spray nozzle, creating problems as having the reaction of the precursor before entering the nozzle and not be able to control the shape. However, when using a long-chain alcohol as the core oil together with relatively gently reactive titanium(Iv) butoxide and titanium(Iv) 2-ethylhexyloxide as precursors, and when tuning their ratio in the oil phase, a high yield could be achieved where most of the oil phase reached the spray nozzle, forming microcapsules. Additional tests showed that the oleyl alcohol/titanium(Iv) butoxide ratio decides the rate of hydrolysis of titanium alkoxides. An optimal fabrication of microcapsules, where most of the reaction between the precursor and water took place in the spray nozzle, was achieved using $20 \mathrm{wt} \%$ titanium(Iv) butoxide in the oil phase, together with $80 \mathrm{wt} \%$ oil. A 1:1 weight ratio gave instead a too fast reaction. The ratio needs to be selected with regard to the aimed product where, in this work, a thin titania shell surrounds the core oil, the latter significantly larger in volume. An excess of the precursor compared to the oil material results in a large fraction of the unreacted precursor.

\section{The active biocide}

As stated in the introduction, microcapsules are mostly formulated and considered in systems where protection or controlled release of an active substance is important. Therefore, we investigated to use the described setup to encapsulate the commonly used biocide 2-n-octyl-4-isothiazolin-3-one, abbreviated OIT. OIT was mixed in the oil phase together with titanium(Iv) butoxide and oleyl alcohol, at different concentrations. To keep a homogeneous one-phase solution of the oil phase, it was found that $50 \mathrm{wt} \%$ of OIT was the maximum concentration still fulfilling this criterion. Four different concentrations of OIT were tested and the differences in the size distribution, fitted with the log-normal 


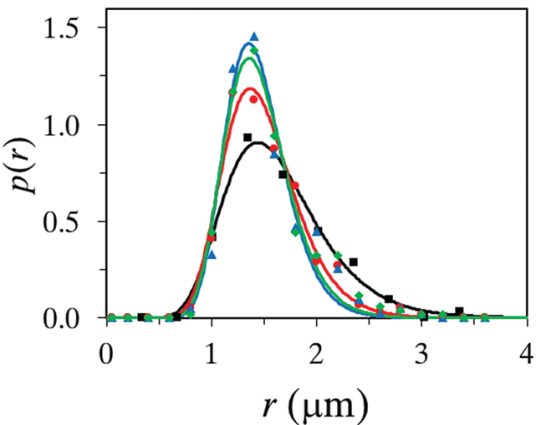

Fig. 6 Size distribution of microcapsules loaded with 0 wt\% (ש), 10 wt\% (O), $25 \mathrm{wt} \%(\boldsymbol{\Delta})$ and $50 \mathrm{wt} \%$ ( OIT.

distribution function, can be seen in Fig. 6. First, we can conclude that microcapsules can be formed using up to $50 \mathrm{wt} \%$ of OIT, together with $20 \mathrm{wt} \%$ titanium(Iv) butoxide and $30 \mathrm{wt} \%$ oleyl alcohol. All recipes up to $50 \mathrm{wt} \%$ OIT produced microcapsules of a few microns in size. However, some differences should be emphasized. The addition of OIT produced microcapsules slightly smaller in size. There is a negligible difference in the size distribution for the samples consisting of 10, 25 and $50 \mathrm{wt} \%$ OIT, respectively. However, formulation with $50 \mathrm{wt} \%$ OIT generated a small but noticeable tail of large, and therefore leaking, microcapsules while such a phenomenon could not be detected at lower concentrations of OIT. Moreover, a small fraction of microcapsules (with $50 \mathrm{wt} \%$ OIT) of different sizes was not perfectly spherical in shape. Fabrication with $10 \mathrm{wt} \%$ and $25 \mathrm{wt} \%$ OIT, respectively, showed high-quality yield of microcapsules, with virtually no aggregates as the formation of titania did not start until reaching the spray nozzle. Since the surface activity of PVA in the water phase and of oleyl alcohol in the oil phase have been discussed as positive parameters, the same might be assumed for OIT. We have in earlier studies shown that OIT appears to possess surfactant-like properties and the OIT-water interfacial tension is as low as $4.5 \mathrm{mN} \mathrm{m}^{-1} .{ }^{13}$ This property could be the reason for the decreasing average size compared to an identical formulation recipe but with no OIT.

\section{The spray nozzle}

All microcapsule formation experiments described above were executed using 2.8 bar $\mathrm{N}_{2}$ as a propellant and this value was chosen since it is the maximum pressure allowed for the spray nozzle in our laboratory. In order to evaluate the effect of propellant pressure for the outcome, pressure values of 1.4 bar and 2.0 bar were also tested, where 1.4 bar is the lower limit for the spray nozzle. The size distribution can be seen in Fig. 7, fitted with the log-normal distribution function. Identical recipes were used in the three different cases; 1 wt $\%$ PVA in the water phase, and an oil phase consisting of the $20 \mathrm{wt} \%$ titanium(Iv) precursor, $70 \mathrm{wt} \%$ oleyl alcohol and $10 \mathrm{wt} \%$ OIT.

All three systems produced a high yield of microcapsules, but with noticeable differences. A nitrogen gas pressure of 1.4 bar generated a significant portion of large capsules $(\sim 10 \mu \mathrm{m})$ which broke early in the collected suspension, and aggregates of broken capsules and unreacted materials were detected.

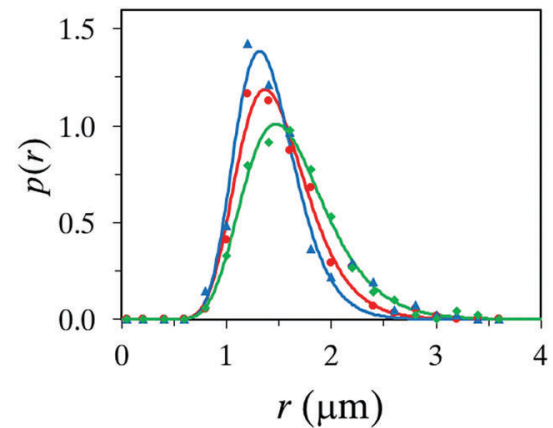

Fig. 7 Size distribution of microcapsules produced with propellant pressures of $2.8(\mathbf{)}), 2.0(\mathbf{\Delta})$, and $1.4(\$)$ bar.

Moreover, a significant amount of the reaction took place at an early stage in the airbrush pen, an equivalent problem that aroused using a non-suitable core oil. A noteworthy improvement was achieved when increasing the pressure to 2.0 bar, forming a larger number of microcapsules with a more narrow size distribution, see Fig. 7. However, a nitrogen pressure of 2.8 bar was found to be preferable. The high pressure produced the highest yield of microcapsules, a narrow size distribution and a setup with condensation of titania that did not initiate until reaching the spray nozzle.

The design of the nozzle is certainly an important feature in the process of creating the intended core-shell structure, since droplets of the oil phase have to be created before the formation of spherical capsules. The hydrolysis of the alkoxide precursor and the condensation of the hydrolyzed species thus formed into titania particles follow a complex sequence of reactions (illustrated in Fig. 5) that occur on a fast time scale and are initiated upon mixing of the two liquids in the airbrush. These reactions thus presumably commence already in or directly after the nozzle. That could also explain the importance of using PVA compared to pure water. If the condensation starts in the nozzle but the process does not finish until in the air or even when collected as suspension, an interfacial stabilizer as PVA seems to be needed. A hypothesis is that a shell only starts to form around the oil droplet, at the interface to the water phase, inside the spray nozzle. In order for complete shell formation, and not only fragments of titania at the interface, the system then needs to be stabilized for some time after the nozzle while the unreacted precursor still in the oil droplet diffuses to the interface. Such a process is then also most probably aided by a surface-active molecule in the oil phase, which we can conclude by the superior results obtained using oleyl alcohol and OIT as a core. However, further experiments are required here to confirm this hypothesis.

\section{Conclusions}

This work shows as a proof of concept for a fast fabrication of oil/ metal oxide core-shell microcapsules using an airbrush pen technique at room temperature without using any toxic solvents or timeconsuming purification steps. The produced shell consists of titania while the composition of the hydrophobic core is rather flexible. 
Several parameters, with respect to both the material properties and the practical formation process, have been studied and tuned to both understand the system and to find the most suitable setup. The pressure of the propellant running the airbrush pen is one parameter to consider, where a higher pressure gave a higher yield of microcapsules. The selection of the core composition is an important factor deciding the quality of the final product. Here, the properties of a long-chain fatty alcohol, in this case oleyl alcohol, indicated the importance of an oil phase consisting of a water insoluble core material and the aiding of its surface activity. At the same time, an encapsulation capacity of up to $50 \mathrm{wt} \%$ of the biocide OIT was achievable. This is interesting in an applied context such as protection of outdoor coatings where OIT is a common choice as an antifouling agent, and where titanium dioxide already can be found as a pigment in the paint matrix. In summary, it can be concluded that a high yield of microcapsules of a narrow size distribution can be achieved in a few minutes using a commercially available airbrush pen, by tuning a few general parameters.

\section{Conflicts of interest}

There are no conflicts of interest to declare.

\section{Acknowledgements}

This work has been carried out partly supported by the Swedish Research Council Formas and the Danish Centre for Energy Materials funded by the Danish Council for Strategic Research. Assistant Professor Tuve Mattson is thanked for generous provision of the filter material. The authors would like to thank the students Viktor Eriksson, Oscar Hjorth, Emil Lukasiewicz, Mate Saric, Erik Walkhed, and Mathias Westerlund for contributions $^{36}$ to the laboratory work.

\section{References}

1 C. Thies, Microencapsulation, Kirk-Othmer Encyclopedia of Chemical Technology, John Wiley \& Sons, Inc., 2005, pp. 438-463.

2 M. Andersson Trojer, Polymeric Core-Shell Particles: Physicochemical Properties and Controlled Release, in Encyclopedia of Surface and Colloid Science, ed. P. Somasundaran, Taylor and Francis, New York, 2013.

3 S. S. Datta, A. Abbaspourrad, E. Amstad, J. Fan, S. H. Kim, M. Romanowsky, H. C. Shum, B. J. Sun, A. S. Utada, M. Windbergs, S. B. Zhou and D. A. Weitz, 25th Anniversary Article: Double Emulsion Templated Solid Microcapsules: Mechanics And Controlled Release, Adv. Mater., 2014, 26(14), 2205-2218.

4 M. H. Lee, K. C. Hribar, T. Brugarolas, N. P. Kamat, J. A. Burdick and D. Lee, Harnessing Interfacial Phenomena to Program the Release Properties of Hollow Microcapsules, Adv. Funct. Mater., 2012, 22(1), 131-138.
5 S. K. Ghosh, Functional Coatings and Microencapsulation: A General Perspective, in Functional Coatings: by Polymer Microencapsulation, ed. S. K. Ghosh, Weinheim, FRG, 2006.

6 C. Dai, B. Wang and H. Zhao, Microencapsulation peptide and protein drugs delivery system, Colloids Surf., B, 2005, 41(2-3), 117-120.

7 M. Li, O. Rouaud and D. Poncelet, Microencapsulation by solvent evaporation: State of the art for process engineering approaches, Int. J. Pharm., 2008, 363(1-2), 26-39.

8 A. Abbaspourrad, N. J. Carroll, S. H. Kim and D. A. Weitz, Polymer Microcapsules with Programmable Active Release, J. Am. Chem. Soc., 2013, 135(20), 7744-7750.

9 A. P. Esser-Kahn, S. A. Odom, N. R. Sottos, S. R. White and J. S. Moore, Triggered Release from Polymer Capsules, Macromolecules, 2011, 44(14), 5539-5553.

10 J. Bergek, M. A. Trojer, H. Uhr and L. Nordstierna, Controlled release of a microencapsulated arduous semihydrophobic active from coatings: Superhydrophilic polyelectrolyte shells as globally rate-determining barriers, J. Controlled Release, 2016, 225, 31-39.

11 M. Andersson Trojer, L. Nordstierna, J. Bergek, H. Blanck, K. Holmberg and M. Nydén, Use of microcapsules as controlled release devices for coatings, Adv. Colloid Interface Sci., 2015, 222, 18-43.

12 M. Andersson Trojer, H. Andersson, Y. Li, J. Borg, K. Holmberg, M. Nyden and L. Nordstierna, Charged microcapsules for controlled release of hydrophobic actives Part III: effect of polyelectrolyte brush- and multilayers on sustained release, Phys. Chem. Chem. Phys., 2013, 15(17), 6456-6466.

13 J. Bergek, M. Andersson Trojer, A. Mok and L. Nordstierna, Controlled release of microencapsulated 2- $n$-octyl-4-isothiazolin3-one from coatings: Effect of microscopic and macroscopic pores, Colloids Surf., A, 2014, 458, 155-167.

14 A. Loxley and B. Vincent, Preparation of poly(methyl methacrylate) microcapsules with liquid cores, J. Colloid Interface Sci., 1998, 208(1), 49-62.

15 M. S. Romero-Cano and B. Vincent, Controlled release of 4-nitroanisole from poly(lactic acid) nanoparticles, J. Controlled Release, 2002, 82(1), 127-135.

16 L. Nordstierna, A. Movahedi and M. Nyden, New Route for Microcapsule Synthesis, J. Dispersion Sci. Technol., 2011, 32(3), 310-311.

17 J. P. Hitchcock, A. L. Tasker, E. A. Baxter, S. Biggs and O. J. Cayre, Long-Term Retention of Small, Volatile Molecular Species within Metallic Microcapsules, ACS Appl. Mater. Interfaces, 2015, 7(27), 14808-14815.

18 K. J. Lin, H. M. Wu, Y. H. Yu, C. Y. Ho, M. H. Wei, F. H. Lu and W. J. Tseng, Preparation of PMMA-Ni core-shell composite particles by electroless plating on polyelectrolytemodified PMMA beads, Appl. Surf. Sci., 2013, 282, 741-745.

19 S. Mandal, M. Sathish, G. Saravanan, K. K. R. Datta, Q. M. Ji, J. P. Hill, H. Abe, I. Honma and K. Ariga, Open-Mouthed Metallic Microcapsules: Exploring Performance Improvements at Agglomeration-Free Interiors, J. Am. Chem. Soc., 2010, 132(41), 14415-14417. 
20 B. Elgh and A. E. C. Palmqvist, A facile low-temperature synthesis of $\mathrm{TiO}_{2}$ nanoparticles with excellent polymorph control, J. Sol-Gel Sci. Technol., 2015, 76(2), 395-401.

21 M. Andersson, L. Österlund, S. Ljungström and A. Palmqvist, Preparation of nanosize anatase and rutile $\mathrm{TiO}_{2}$ by hydrothermal treatment of microemulsions and their activity for photocatalytic wet oxidation of phenol, J. Phys. Chem. B, 2002, 106(41), 10674-10679.

22 T. C. Chou, T. R. Ling, M. C. Yang and C. C. Liu, Micro and nano scale metal oxide hollow particles produced by spray precipitation in a liquid-liquid system, Mater. Sci. Eng., A, 2003, 359(1-2), 24-30.

23 M. Iida, T. Sasaki and M. Watanabe, Titanium dioxide hollow microspheres with an extremely thin shell, Chem. Mater., 1998, 10(12), 3780-3782.

24 L. X. Chai, X. D. Wang and D. Z. Wu, Development of bifunctional microencapsulated phase change materials with crystalline titanium dioxide shell for latent-heat storage and photocatalytic effectiveness, Appl. Energy, 2015, 138, 661-674.

25 A. M. Collins, C. Spickermann and S. Mann, Synthesis of titania hollow microspheres using non-aqueous emulsions, J. Mater. Chem., 2003, 13(5), 1112-1114.

26 L. Coulthwaite, K. Bayley, C. Liauw, G. Craig and J. Verran, The effect of free and encapsulated OIT on the biodeterioration of plasticised PVC during burial in soil for 20 months, Int. Biodeterior. Biodegrad., 2005, 56(2), 86-93.

27 M. Edge, N. S. Allen, D. Turner, J. Robinson and K. Seal, The enhanced performance of biocidal additives in paints and coatings, Prog. Org. Coat., 2001, 43(1-3), 10-17.
28 J. Livage, M. Henry and C. Sanchez, Sol-gel chemistry of transition metal oxides, Prog. Solid State Chem., 1988, 18(4), 259-341.

29 G. W. Scherer and C. J. Brinker, Sol-Gel Science, Academic Press, London, 1990.

30 J. Goodman, Computer Software Review: Reaxys, J. Chem. Inf. Model., 2009, 49(12), 2897-2898.

31 E. E. Bolton, Y. Wang, P. A. Thiessen and S. H. Bryant, Chapter 12 - PubChem: Integrated Platform of Small Molecules and Biological Activities, Annu. Rep. Comput. Chem., 2008, 4, 217-241.

32 R. M. Stephenson, Mutual solubilities: water-ketones, waterethers, and water-gasoline-alcohols, J. Chem. Eng. Data, 1992, 37(1), 80-95.

33 D. J. Letinski, M. J. Connelly, D. R. Peterson and T. F. Parkerton, Slow-stir water solubility measurements of selected alcohols and diesters, Chemosphere, 2002, 48(3), 257-265.

34 R. Stephenson and J. Stuart, Mutual binary solubilities: water-alcohols and water-esters, J. Chem. Eng. Data, 1986, 31(1), 56-70.

35 J. Petermann, Der Einfluß monomolekularer Filme auf die Kinetik der Gasabsorption in wäßrige Lösungen, Ber. BunsenGes. Phys. Chem., 1977, 81(7), 649-656.

36 V. Eriksson, O. Hjorth, E. Lukasiewicz, M. Saric, E. Walkhed and $\mathbf{M}$. Westerlund, Formulering av titandioxidmikrokapslar genom en sprayteknik, in Instutionen för Kemi-och kemieknik, Chalmers tekniska högskola, Chalmers tekniska högskola, Göteborg, 2017. 